\title{
Direction finding of bistatic MIMO radar based on quantum-inspired grey wolf optimization in the impulse noise
}

\author{
Hongyuan Gao* D, Jia Li and Ming Diao
}

\begin{abstract}
A novel direction-finding method is proposed for bistatic multiple-input-multiple-output (MIMO) radar in the impulse noise in this paper. The method has the capacity to suppress the impulse noise by means of infinite norm normalization and can obtain better performance for direction finding via the weighted signal subspace fitting algorithm. To solve the objective function of this method, we devise a quantum-inspired grey wolf optimization algorithm to acquire the global optimal solution. The proposed method based on QGWO can resolve the direction-finding difficulties of bistatic MIMO radar. Monte-Carlo experiments have confirmed the robustness and superiority of the proposed method for locating independent and coherent sources with a small number of snapshots in the impulse noise compared with some existing direction-finding methods in a series of scenarios. In addition, we present the Cramér-Rao bound (CRB) of angle estimation for bistatic MIMO radar in the impulse noise, which generalizes the Gaussian CRB for performance analysis.
\end{abstract}

Keywords: Bistatic MIMO radar, Direction finding, Impulse noise, Infinite norm normalization, Weighted signal subspace fitting, Quantum-inspired grey wolf optimization, Cramér-Rao bound

\section{Introduction}

Recently, multiple-input-multiple-output (MIMO) radar has caused extensive concern because of its better parameter estimation performance compared with phased array radar [1]. Overall, there are two types of MIMO radar according to the radar configurations: statistical MIMO radar and colocated MIMO radar. The first type, whose antennas are widely separated in both the transmitting and receiving arrays, can achieve high resolution via the spatial diversity gain in multiple channels $[2,3]$. The second type, whose antennas are closely located in the transmitting and receiving arrays, can achieve a coherent processing gain $[4,5]$. Furthermore, the transmitters and receivers of the monostatic colocated MIMO radar are closely located, which causes the result that the direction of departure (DOD) of a target is the same as the direction of arrival (DOA) of the target. For the bistatic colocated MIMO radar, the transmitters are far from the

*Correspondence: gaohongyuan@hrbeu.edu.cn

College of Information and Communication Engineering, Harbin Engineering University, Nantong Street, Harbin, China receivers, which brings about different DOD and DOA. In this paper, we consider the bistatic colocated MIMO radar to study.

Direction finding is a major issue of interest in array signal processing for MIMO radar. In this context, this issue has drawn attention from researchers in recent years. The quaternion theory was applied in direction finding for bistatic MIMO radar by means of singular value decomposition and root multiple signal classification (Root-MUSIC) in [6]. An ESPRIT-based algorithm was presented via the banded symmetric and Toeplitz matrices for direction finding of bistatic MIMO radar in [7]. The unitary dual-resolution ESPRIT algorithm was proposed for direction finding of bistatic MIMO radar without extra pairing in [8]. Moreover, the maximum likelihood direction finding method for bistatic MIMO radar was proposed in [9].

Overall, these methods have been studied for additive Gaussian noise, but their performance can deteriorate in the presence of non-Gaussian noise. The impulsivity is a major characteristic of the non-Gaussian noise. In the real world, the atmospheric noise, switching transients, 
and lightning are the typical impulse noises, which are characterized by significant peak values. In this sense, the symmetric $\alpha$-stable ( $\mathrm{S} \alpha \mathrm{S})$ distribution, which generalizes the Gaussian distribution, is an appropriate model to describe the impulse noise $[10,11]$.

However, there are no second and higher-order moments in the $\mathrm{S} \alpha \mathrm{S}$ distribution, and the conventional direction-finding methods based on the second and higher-order moments are not able to work on the impulse noise. In this case, fractional low-order moment (FLOM) was applied to suppress the impulse noise, and the FLOMESPRIT method was proposed for direction finding of bistatic MIMO radar in [12]. A FLOM-unitary ESPRIT method for direction finding of bistatic MIMO radar was proposed in the impulse noise in [13]. In [14], the FLOM-MUSIC method was presented for direction finding of MIMO radar in the impulse noise, then an infinite norm (IN) normalization MUSIC method was proposed to avoid obtaining prior knowledge about characteristic exponent to achieve effective noise suppression. Compared with fractional low-order moment, we can improve the performance of suppressing the impulse noise via infinite norm normalization. The weighted signal subspace fitting (WSSF) algorithm [15] can achieve higher accuracy in comparison to these subspace-based algorithms. But under the background of impulse noise, the basic WSSF method can not guarantee the performance. In order to suppress the impulse noise, we introduced the IN normalization to WSSF method for the first time and proposed the IN-WSSF method for the direction finding of the bistatic MIMO radar. Moreover, the proposed IN-WSSF method is insensitive to the signal-to-noise ratio (SNR) and snapshots.

Nevertheless, it is difficult to implement the IN-WSSF method, because it requires the maximization of the cost function, which is high-dimensional, nonlinear, and multimodal. These characteristics motivate the development of a series of alternative optimization algorithms, one of which is the intelligence algorithm. They have the capacity to reduce the computational complexity and accelerate the speed of convergence when solving the IN-WSSF function to perform direction finding.

Intelligence algorithms, such as particle swarm optimization (PSO) [16], artificial bee colony (ABC) [17], harmony search (HS) [18], and cuckoo search (CS) [19], have been remarkably popular over the past two decades, and they have been employed in a variety of domains $[20,21]$ in a black-box style because of their simplicity and derivation-free mechanism. However, some of them can be easily trapped in a local optimum or not rapidly converge to the global optimum, both of which are obstacles for engineering problems, especially in the directionfinding issue. Hence, we propose a quantum-inspired grey wolf optimization (QGWO) algorithm, which is inspired by quantum computing [22] and the social hierarchy of grey wolves [23]. Additionally, the proposed QGWO algorithm can effectively avoid converging to local optima and accelerate the speed of convergence compared with the original grey wolf optimization (GWO) [23]. Then, the global optimal solution of the proposed IN-WSSF method can be achieved by the QGWO, which is referred to as the QGWO-IN-WSSF method for short.

For the purpose of analyzing the performance of the QGWO-IN-WSSF method, we first derive a general expression of the Cramér-Rao bound (CRB) of angle estimation for bistatic MIMO radar in the impulse noise. Then, our work is pushed forward in testing the performance of the proposed QGWO-IN-WSSF method in different direction-finding scenarios, including a number of snapshots and sources. Monte-Carlo experiments provide the evidence that the proposed QGWO-IN-WSSF method has obtained the expected performance in different direction-finding scenarios.

To summarize, the major contributions of this paper can be briefly listed in the following:

1. A novel direction-finding method of bistatic MIMO radar is addressed in the impulse noise, which can effectively suppress the impulse noise and achieve the desired performance with a small number of snapshots.

2. The proposed direction-finding method of bistatic MIMO radar can locate the coherent sources without extra preprocessing techniques.

3. A novel iterative optimization algorithm, the QGWO, is devised to solve the continuous optimization problem of the IN-WSSF method.

4. A general expression of the CRB, as the generalization of the Gaussian CRB, is presented for direction finding of bistatic MIMO radar in the impulse noise.

5. The Cauchy-Gaussian mixture (CGM) model is adopted for the approximation of the probability distribution function (PDF) of the $S \alpha \mathrm{S}$ distribution in order to obtain the CRB.

The remainder of this paper is organized as follows. In Section 2, the direction-finding model of bistatic MIMO radar in the impulse noise and the IN-WSSF method are addressed. In Section 3, we provide the detailed description of QGWO, and then, the QGWO is applied to solve the cost function of the IN-WSSF method. In Section 4, the CRB for direction finding of bistatic MIMO radar in the impulse noise is derived for performance analysis. In Section 5, the performance of QGWO-IN-WSSF is examined in comparison to several existing directionfinding methods in some scenarios, and some important evaluations and remarks are offered via the simulations. Finally, Section 6 concludes the paper and presents the subsequent research interests.

The following notations are used in this paper. Matrices and vectors are represented by bold uppercase and 
bold lowercase characters, respectively. Vectors are in column orientation by default, and superscripts $(\cdot)^{T},(\cdot)^{*}$, and $(\cdot)^{H}$ denote the transpose, the conjugate, and the conjugate transpose. $[\cdot]_{i j}$ denotes the $i$ th row and the $j$ th column element of the matrix. Furthermore, $\operatorname{tr}(\cdot), \oplus, \otimes, \operatorname{diag}(\cdot)$, $E[\cdot], \operatorname{Re}(\cdot), \operatorname{Im}(\cdot)$, and $|\cdot|$ stand for the trace of the matrix, the Hadamard product, the Kronecker product, the diagonal operator, the expectation, the real part, the imaginary part, and the absolute value operator, respectively. Finally, $\|\cdot\|^{2}$ represents the normalized norm of the vector.

\section{Direction-finding model for bistatic MIMO radar in the impulse noise}

We assume that there are $M$ transmitting antennas and $N$ receiving antennas in bistatic MIMO radar and both sides are a uniform linear array (ULA). The transmitting side can transmit $M$ temporally orthogonal signals at the same time, and they have the same center frequency and bandwidth. Here, $\mathbf{s}(k)=\left[s_{1}(k), s_{2}(k), \ldots, s_{M}(k)\right]^{T}$ denotes the transmitting signal vector, and its elements satisfy the following orthogonality:

$$
\frac{1}{K} \sum_{k=1}^{K} s_{i}(k) s_{j}^{*}(k)=\left\{\begin{array}{l}
1, i=j \\
0, i \neq j
\end{array},\right.
$$

where $s_{i}(k)$ and $s_{j}(k)$ denote the signals transmitted from the $i$ th and $j$ th transmitting elements, respectively, $i=1,2, \ldots, M, j=1,2, \ldots, M, k=1,2, \ldots, K$, and $K$ represents the maximum number of snapshots.

We consider $P$ targets that appear in the far field. $\theta=\left[\theta_{1}, \theta_{2}, \ldots, \theta_{P}\right]^{T}, \boldsymbol{\varphi}=\left[\varphi_{1}, \varphi_{2}, \ldots, \varphi_{P}\right]^{T}$, and $\boldsymbol{\beta}=\left[\beta_{1}, \beta_{2}, \ldots, \beta_{P}\right]^{T}$ represent the DOD vector, DOA vector, and complex reflection coefficient vector of the targets, respectively. Then, the received signal vector can be expressed as follows:

$$
\mathbf{r}(k)=\mathbf{A}_{\mathrm{r}}(\boldsymbol{\varphi}) \Lambda_{\beta} \mathbf{A}_{\mathrm{t}}^{T}(\theta) \mathbf{s}(k)+\tilde{\mathbf{n}}(k),
$$

where $k=1,2, \ldots, K, \mathbf{A}_{\mathrm{r}}(\boldsymbol{\varphi})=\left[\mathbf{a}_{\mathrm{r}}\left(\varphi_{1}\right), \mathbf{a}_{\mathrm{r}}\left(\varphi_{2}\right), \ldots\right.$, $\left.\mathbf{a}_{\mathrm{r}}\left(\varphi_{P}\right)\right]$ represents the $N \times P$ receiving array manifold, $\mathbf{A}_{\mathrm{t}}(\boldsymbol{\theta})=\left[\mathbf{a}_{\mathrm{t}}\left(\theta_{1}\right), \mathbf{a}_{\mathrm{t}}\left(\theta_{2}\right), \ldots, \mathbf{a}_{\mathrm{t}}\left(\theta_{P}\right)\right]$ represents the $M \times P$ transmitting array manifold, $\mathbf{a}_{\mathbf{r}}\left(\varphi_{p}\right)(p=1,2, \ldots, P)$ is the $N \times 1$ receiving steering vector of $\varphi_{p}, \mathbf{a}_{\mathrm{t}}\left(\theta_{p}\right)(p=$ $1,2, \ldots, P)$ is the $M \times 1$ transmitting steering vector of $\theta_{p}, \Lambda_{\beta}=\operatorname{diag}(\beta)$ is the diagonal matrix constituted by $\beta$ which obeys the standard normal distribution, and $\tilde{\mathbf{n}}(k)$ denotes the $N \times 1$ noise vector, which is assumed to be impulsive and modeled by standard $S \alpha S$ distribution in this paper.

For the purpose of separating the orthogonal components of the transmitting signals, matched filters are employed at the receiving side. Then, the output after matched filtering is given by

$$
\begin{aligned}
\mathbf{y}(k) & =\sum_{p=1}^{P} \beta_{p} \mathbf{a}_{\mathbf{t}}\left(\theta_{p}\right) \otimes \mathbf{a}_{\mathbf{r}}\left(\varphi_{p}\right)+\mathbf{n}(k) \\
& =\mathbf{C}(\theta, \varphi) \beta(k)+\mathbf{n}(k),
\end{aligned}
$$

where $\mathbf{C}(\boldsymbol{\theta}, \boldsymbol{\varphi})=\left[\mathbf{a}_{\mathrm{t}}\left(\theta_{1}\right) \otimes \mathbf{a}_{\mathrm{r}}\left(\varphi_{1}\right), \mathbf{a}_{\mathrm{t}}\left(\theta_{2}\right) \otimes \mathbf{a}_{\mathrm{r}}\left(\varphi_{2}\right), \ldots\right.$, $\left.\mathbf{a}_{\mathrm{t}}\left(\theta_{P}\right) \otimes \mathbf{a}_{\mathrm{r}}\left(\varphi_{P}\right)\right]$ is the $M N \times P$ transmitting-receiving array manifold, and $\mathbf{n}(k)$ denotes the $M N \times 1$ complex impulse noise vector.

Then, the estimation of the covariance matrix for $\mathbf{y}(k)$ can be obtained while considering the finite snapshots as follows:

$$
\hat{\mathbf{R}}_{\mathrm{y}}=\frac{1}{K} \sum_{k=1}^{K} \mathbf{y}(k) \mathbf{y}^{H}(k),
$$

Hence, the eigendecomposition of $\hat{\mathbf{R}}_{\mathrm{y}}$ is given by

$$
\hat{\mathbf{R}}_{\mathrm{y}}=\hat{\mathbf{U}}_{\mathrm{s}} \hat{\boldsymbol{\Lambda}}_{\mathrm{s}} \hat{\mathbf{U}}_{\mathrm{s}}^{H}+\hat{\mathbf{U}}_{\mathrm{n}} \hat{\boldsymbol{\Lambda}}_{\mathrm{n}} \hat{\mathbf{U}}_{\mathrm{n}}^{H},
$$

where $\hat{\boldsymbol{\Lambda}}_{\mathrm{s}}$ and $\hat{\boldsymbol{\Lambda}}_{\mathrm{n}}$ denote the diagonal matrices that are constructed by the eigenvalues of the signal-subspace and noise-subspace, respectively, and the column vectors of matrices $\hat{\mathbf{U}}_{\mathrm{s}}$ and $\hat{\mathbf{U}}_{\mathrm{n}}$ represent the eigenvectors of the signal-subspace and noise-subspace, respectively.

According to weighted signal subspace fitting, the estimation of $\{\theta, \varphi\}$ can be achieved via the following function (the characteristic exponent $\alpha=2$ ) [15]:

$$
\{\hat{\theta}, \hat{\varphi}\}=\arg \max _{\boldsymbol{\theta}, \boldsymbol{\varphi}} \operatorname{tr}\left[\mathbf{P}_{\mathbf{C}(\boldsymbol{\theta}, \boldsymbol{\varphi})} \hat{\mathbf{U}}_{\mathrm{s}} \hat{\mathbf{W}}_{\mathbf{\mathbf { U }}} \hat{\mathbf{s}}^{H}\right],
$$

where $\mathbf{P}_{\mathbf{C}(\boldsymbol{\theta}, \boldsymbol{\varphi})}=\mathbf{C}(\boldsymbol{\theta}, \boldsymbol{\varphi})\left[\mathbf{C}^{H}(\boldsymbol{\theta}, \boldsymbol{\varphi}) \mathbf{C}(\boldsymbol{\theta}, \boldsymbol{\varphi})\right]^{-1} \mathbf{C}^{H}(\boldsymbol{\theta}, \boldsymbol{\varphi})$ represents the projection matrix of $\mathbf{C}(\theta, \varphi), \hat{\mathbf{W}}$ is the weighted matrix, and the optimal weighted matrix is given by

$$
\hat{\mathbf{W}}_{\mathrm{opt}}=\left(\hat{\boldsymbol{\Lambda}}_{\mathrm{s}}-\hat{\sigma}_{\mathrm{n}}^{2} \mathbf{I}\right)^{2} \hat{\boldsymbol{\Lambda}}_{\mathrm{s}}^{-1},
$$

where $\hat{\sigma}_{\mathrm{n}}^{2}$ represents the estimation of the noise variance, and I represents the identity matrix.

Because there is no second-order moment when the characteristic exponent $\alpha<2$, weighted signal subspace fitting algorithm based on the second-order moment is not able to work on the impulse noise. Thus, we utilize the infinite norm normalization method to deal with the problem, which does not need any prior knowledge about characteristic exponent and is able to improve the performance of suppressing the impulse noise compared with the FLOM. The normalized data can be described as:

$$
\mathbf{z}(k)=\frac{\mathbf{y}(k)}{\max \left\{\left|y_{1}(k)\right|,\left|y_{2}(k)\right|, \ldots,\left|y_{M N}(k)\right|\right\}},
$$

where $\mathbf{y}(k)=\left[y_{1}(k), y_{2}(k), \ldots, y_{M N}(k)\right]^{T}$.

Remark. The weighted signal covariance matrix $\mathbf{R}_{\mathrm{z}}=$ $E\left[\mathbf{z}(k) \mathbf{z}^{H}(k)\right]$ is bounded in the impulse noise. 
Theoretical analysis demonstrates that the weighted signal covariance matrix $\mathbf{R}_{\mathrm{z}}$ is finite and the cross-correlation is also zero. Then, the estimation of weighted signal covariance matrix can be given by:

$$
\hat{\mathbf{R}}_{\mathbf{z}}=\frac{1}{K} \sum_{k=1}^{K} \mathbf{z}(k) \mathbf{z}^{H}(k) .
$$

In this case, we can obtain the signal-subspace and noise-subspace by the eigendecomposition then achieve the estimation of $\{\theta, \varphi\}$ according to weighted signal subspace fitting algorithm in the impulse noise.

To summarize, weighted signal subspace fitting algorithm based on infinite norm normalization is suitable for Gaussian noise $(\alpha=2)$ and impulsive noise $(\alpha<2)$.

In addition, the proposed method can be applied to other more complicated array structures. Although the array manifold of complicated array structure is different from that of the ULA, the style of corresponding direction-finding function is similar to the ULA. Based on this, the proposed method can be expanded to realize the sources estimation of some complicated arrays.

\section{Direction-finding method based on quantum-inspired grey wolf optimization for bistatic MIMO radar}

\subsection{Quantum-inspired grey wolf optimization}

Grey wolf optimization (GWO) algorithm is inspired by the social hierarchy of grey wolves. Grey wolves usually live in a pack, and they have a very strict social dominant hierarchy. The head grey wolf at the first level of hierarchy is called epsilon $(\varepsilon)$, and $\varepsilon$ is the leader of grey wolf swarm. Its function is to make decisions about hunting, habitat, food distribution, and so on. The grey wolf at the second level of hierarchy is called eta $(\eta)$. When $\varepsilon$ is missing from the grey wolves, $\eta$ will replace $\varepsilon$ as the new head grey wolf. The grey wolf at the third level of hierarchy is rho $(\rho), \rho$ follows the instructions of $\varepsilon, \eta$, the old $\varepsilon$ and old $\eta$ would also fall into this level. The remaining grey wolves at the final level are responsible for balancing the internal relations of the grey wolf swarm. The principle of GWO algorithm is simple, the parameters needed to be adjusted are few, and it is easy to be realized. But the basic GWO algorithm is easy to fall into local optimum, which leads to the failure of finding the optimal solution. Besides, the convergence value is not accurate enough for the complex continuous optimization problems.

According to the theory of GWO algorithm and quantum computation, the QGWO algorithm is proposed in this paper. In the QGWO algorithm, we design the entirely new equations compared with the GWO, which can improve the search ability and overcome the drawbacks of GWO algorithm.
There is a grey wolf swarm with $Q$ grey wolves, each of which possesses its own position. Thus, we define the $q$ th grey wolf's position at the $t$ th iteration as $\tilde{\mathbf{x}}_{q}^{t}=$ $\left[\tilde{x}_{q, 1}^{t}, \tilde{x}_{q, 2}^{t}, \ldots, \tilde{x}_{q, B}^{t}\right](q=1,2, \ldots, Q)$ mapped by the quantum position $\mathbf{x}_{q}^{t}=\left[x_{q, 1}^{t}, x_{q, 2}^{t}, \ldots, x_{q, B}^{t}\right]$, where $0 \leq x_{q, b}^{t} \leq$ $1(b=1,2, \ldots, B)[24]$. Accordingly, the mapping function is defined as follows:

$$
\tilde{x}_{q, b}^{t}=\tilde{x}_{b}^{\text {low }}+x_{q, b}^{t} \cdot\left(\tilde{x}_{b}^{\text {high }}-\tilde{x}_{b}^{\text {low }}\right),
$$

where $\tilde{x}_{q, b}^{t} \in\left[\tilde{x}_{b}^{\text {low }}, \tilde{x}_{b}^{\text {high }}\right], \tilde{x}_{b}^{\text {low }}$ denotes the $b$ th dimensional lower bound, and $\tilde{x}_{b}^{\text {high }}$ denotes the $b$ th dimensional upper bound.

The position of each grey wolf represents a potential solution with $B$-dimension. Thus, the quality of a potential solution can be evaluated through the fitness function $F\left(\tilde{\mathbf{x}}_{q}^{t}\right)$, whose value denotes the fitness of $\tilde{\mathbf{x}}_{q}^{t}$.

According to the feature of social hierarchy in the grey wolf swarm, we define that three grey wolves corresponding to the first three best quantum positions obtained so far are the epsilon $(\varepsilon)$, eta $(\eta)$, and rho $(\rho)$, respectively. In addition, the omega $(\omega)$ denotes one of the remaining grey wolves. To hunt the prey (locate the potential global optimum), we update the quantum positions of the omegas through the epsilon, eta, and rho during the iterations. In this sense, there are two types of novel quantum position updating strategies in the proposed QGWO. The updating equations of the first strategy are proposed by using a simplified simulated quantum rotation gate as follows:

$$
\begin{gathered}
\dot{\delta}_{q, b}^{t+1}=\lambda_{1} \cdot\left|c_{1} \cdot x_{b}^{\varepsilon}-x_{q, b}^{t}\right|, \\
\ddot{\delta}_{q, b}^{t+1}=\lambda_{2} \cdot\left|c_{2} \cdot x_{b}^{\eta}-x_{q, b}^{t}\right|, \\
\dddot{\delta}_{q, b}^{t+1}=\lambda_{3} \cdot\left|c_{3} \cdot x_{b}^{\rho}-x_{q, b}^{t}\right|, \\
\dot{x}_{q, b}^{t+1}=\left|x_{b}^{\varepsilon} \times \cos \left(\dot{\delta}_{q, b}^{t+1}\right)+\sqrt{1-\left(x_{b}^{\varepsilon}\right)^{2}} \times \sin \left(\dot{\delta}_{q, b}^{t+1}\right)\right|, \\
\ddot{x}_{q, b}^{t+1}=\left|x_{b}^{\eta} \times \cos \left(\ddot{\delta}_{q, b}^{t+1}\right)+\sqrt{1-\left(x_{b}^{\eta}\right)^{2}} \times \sin \left(\ddot{\delta}_{q, b}^{t+1}\right)\right|, \\
\dddot{x}_{q, b}^{t+1}=\left|x_{b}^{\rho} \times \cos \left(\dddot{\delta}_{q, b}^{t+1}\right)+\sqrt{1-\left(x_{b}^{\rho}\right)^{2}} \times \sin \left(\dddot{\delta}_{q, b}^{t+1}\right)\right|, \\
x_{q, b}^{t+1}=\frac{\dot{x}_{q, b}^{t+1}+\ddot{x}_{q, b}^{t+1}+\dddot{x}_{q, b}^{t+1}}{3}
\end{gathered}
$$

where $x_{b}^{\varepsilon}, x_{b}^{\eta}$, and $x_{b}^{\rho}$ denote the $b$ th dimension of the quantum positions of $\varepsilon, \eta$, and $\rho$, respectively, $\dot{\delta}_{q, b}^{t+1}, \ddot{\delta}_{q, b}^{t+1}$, and $\dddot{\delta}_{q, b}^{t+1}$ represent the quantum rotation angles of $\varepsilon$, $\eta$, and $\rho$, respectively, $t$ denotes the iteration number, 
$c_{\tau}=2 \cdot r_{1}, \lambda_{\tau}=\left(2 \cdot r_{2}-1\right) \cdot \mu, \tau=1,2,3, r_{1}$ and $r_{2}$ are the uniform random numbers in [0,1], and $\mu$ decreases linearly from 2 to 0 during the iterations.

For the second quantum position updating strategy, the updating equations are adjusted by altering the size and direction of the searching as follows:

$$
\begin{gathered}
\delta_{q, b}^{t+1}=r_{3} \cdot\left(x_{b}^{\varepsilon}-x_{q, b}^{t}\right)+r_{4} \cdot\left(\bar{x}_{b}^{t}-x_{q, b}^{t}\right), \\
x_{q, b}^{t+1}=\left|x_{q, b}^{t} \times \cos \left(\delta_{q, b}^{t+1}\right)+\sqrt{1-\left(x_{q, b}^{t}\right)^{2}} \times \sin \left(\delta_{q, b}^{t+1}\right)\right|,
\end{gathered}
$$

where $r_{3}$ denotes a uniform random number in $[0,1], r_{4}$ represents a random number that obeys the standardized normal distribution, $\delta_{q, b}^{t+1}$ denotes the quantum rotation angle, and $\bar{x}_{b}^{t}=(1 / Q) \sum_{q=1}^{Q} x_{q, b}^{t}$ is the $b$ th dimension of the average value $\overline{\mathbf{x}}^{t}=\left[\bar{x}_{1}^{t}, \bar{x}_{2}^{t}, \ldots, \bar{x}_{B}^{t}\right]$ of $Q$ quantum positions.

\subsection{Computational complexity analysis of the QGWO algorithm}

As described in Section 3.1, the QGWO algorithm updates the quantum rotation angles in all dimensions of all grey wolves at each iteration, and the corresponding complexity is $O(Q B)$, where $Q$ is the population size of grey wolf swarm and $B$ denotes the dimension of the optimization problem. The quantum position of each grey wolf is updated by the quantum rotation angle, and the position of the grey wolf can be obtained by mapping from the quantum position, with the computational complexity $O(2 Q B)$. After that, we need to calculate the corresponding fitness of the updated positions with complexity $O(Q)$. By means of the greedy selection, the current epsilon $(\varepsilon)$, eta $(\eta)$, and rho $(\rho)$ are updated at each iteration, and the complexity is $O(Q)$.

When the number of terminated iterations is $t$, the computational complexity of the QGWO algorithm is $O(t(3 Q B+2 Q))$.

\subsection{QGWO-IN-WSSF method for bistatic MIMO radar}

For the QGWO, each dimension of the initial quantum positions is randomly created in $[0,1]$. For the direction finding problem of this paper, the fitness function should be defined as follows:

$$
F\left(\tilde{\mathbf{x}}_{q}^{t}\right)=\operatorname{tr}\left[\mathbf{P}_{\mathbf{C}\left(\tilde{\mathbf{x}}_{q}^{t}\right)} \hat{\mathbf{U}}_{\mathrm{s}} \hat{\mathbf{W}}_{\mathbf{U}_{\mathrm{s}}^{H}}^{H}\right]
$$

where the grey wolf's position $\tilde{\mathbf{x}}_{q}^{t}=\left[\tilde{x}_{q, 1}^{t}, \tilde{x}_{q, 2}^{t}, \ldots, \tilde{x}_{q, B}^{t}\right]$ corresponds to a set of estimation values of angles, $B=2 P$, and $P$ denotes the number of targets.

As described above, the issue can be converted into a continuous optimization problem. In the process of implementing QGWO, two types of quantum position updating strategies are repeatedly applied until the number of iterations has been reached. To summarize, the QGWO-IN-WSSF method is outlined as follows:

Step 1: Initialize the arguments for the QGWO: the population size $Q, \mu, \lambda_{\tau}, c_{\tau}(\tau=1,2,3)$, and the number of iterations.

Step 2: Randomly create the initial quantum positions of $Q$ grey wolves in $[0,1]$.

Step 3: Compute the fitness of the initial positions mapping from the quantum positions of grey wolves. According to the fitness values, select the first three best quantum positions as the initial epsilon, eta, and rho.

Step 4: Update the current quantum positions of grey wolves by means of these two strategies with the probability of $50 \%$.

Step 5: Calculate corresponding fitness of the updated positions mapping from the quantum positions of grey wolves.

Step 6: According to the new fitness values, update the current epsilon, eta, and rho by greedy selection and update the arguments $\mu, \lambda_{\tau}, c_{\tau}$.

Step 7: Examine the end criterion: if satisfied, break up and output the ultimate mapping position of epsilon which corresponds to a set of global optimal estimation values of angles; otherwise, go back to step 4 .

\section{Performance analysis for direction finding of bistatic MIMO radar in the impulse noise}

\subsection{Basic definitions in the impulse noise model}

Because there is a heavy tail in the PDF of the impulse noise, the $\mathrm{S} \alpha \mathrm{S}$ distribution with zero-location is an appropriate model to describe it, and its characteristic function can be represented as follows:

$$
\phi(w)=\mathrm{e}^{-\gamma^{\alpha}|w|^{\alpha}},
$$

where $0<\alpha \leq 2$ denotes the characteristic exponent, $\gamma>0$ represents the scale, and $\gamma^{2}$ resembles the variance for the Gaussian distribution.

The tail of the PDF for the $\mathrm{S} \alpha \mathrm{S}$ distribution is depicted via $\alpha$. In addition, a small value of $\alpha$ leads to a strong impulsivity [10]. However, the SNR is usually insignificant for the $\mathrm{S} \alpha \mathrm{S}$ distribution because of the non-existence of its second and higher order moments. Therefore, a generalized SNR (GSNR) is defined as follows [25]:

$$
\operatorname{GSNR}=10 \lg \left\{\frac{E\left[\|\mathbf{s}(k)\|^{2}\right]}{\gamma^{\alpha}}\right\},
$$

where $E\left[\|\mathbf{s}(k)\|^{2}\right]$ is the average power of the signals.

\subsection{Cramér-Rao bound in the impulse noise}

To verify the performance of the direction-finding methods, the CRB is a useful mathematical tool. Under the 
background of Gaussian noise, the CRB [26] has been discussed for many different direction-finding methods [27]. But the related researches for the CRB in the impulse noise are few.

We will derive the CRB of the estimated parameters for bistatic MIMO radar in the impulse noise to analyze the performance of direction finding in this section. First, we consider the following direction-finding model:

$$
\begin{aligned}
\mathbf{y}(k) & =\mathbf{C}(\theta, \varphi) \boldsymbol{\beta}(k)+\mathbf{n}(k) \\
& =\mathbf{g}(\boldsymbol{\Gamma}, k)+\mathbf{h}(k),
\end{aligned}
$$

where $\mathbf{g}(\boldsymbol{\Gamma}, k)$ and $\mathbf{h}(k)$ denote the signal component and noise component, respectively, a complex quantity $v=$ $\bar{v}+\mathrm{j} \tilde{v}, \boldsymbol{\Gamma}=[\theta, \varphi, \overline{\boldsymbol{\beta}}(1), \overline{\boldsymbol{\beta}}(2), \ldots, \overline{\boldsymbol{\beta}}(K), \tilde{\boldsymbol{\beta}}(1), \tilde{\boldsymbol{\beta}}(2), \ldots$, $\tilde{\boldsymbol{\beta}}(K)], \mathbf{h}(k)=\left[h_{1}(k), h_{2}(k), \ldots, h_{M N}(k)\right]^{T}$, the element $h_{l}(k)=\bar{h}_{l}(k)+\mathrm{j} \tilde{h}_{l}(k)(l=1,2, \ldots, M N), k=1,2, \ldots, K$, and $f_{h}(\bar{h}, \tilde{h})$ denotes its PDF with parameter $\Omega$, which satisfies the regularity conditions [28].

In general, the CRB of $\Gamma$ is a major issue of interest, and thus, the CRB can be represented as follows:

$$
\operatorname{CRB}\left(\hat{\Gamma}_{i}\right) \geq\left[\mathbf{J}_{\mathbf{\Gamma}}^{-1}\right]_{i i},
$$

where $\hat{\Gamma}_{i}$ is the estimation of an element $\Gamma_{i}$ in $\boldsymbol{\Gamma}$, and $\mathbf{J}_{\boldsymbol{\Gamma}}$ is the Fisher information matrix (FIM) of $\Gamma$ whose element is given by:

$$
J_{i j}=E\left[\frac{\partial \ln f_{\mathbf{Y}}(\mathbf{Y})}{\partial \Gamma_{i}} \frac{\partial \ln f_{\mathbf{Y}}(\mathbf{Y})}{\partial \Gamma_{j}}\right],
$$

where $\mathbf{Y}=[\mathbf{y}(1), \mathbf{y}(2), \ldots, \mathbf{y}(K)]$, whose PDF can be represented as

$$
f_{\mathbf{Y}}(\mathbf{Y})=\prod_{k=1}^{K} \prod_{l=1}^{M N} f_{h}\left[\bar{y}_{l}(k)-\bar{g}_{l}(\boldsymbol{\Gamma}, k), \tilde{y}_{l}(k)-\tilde{g}_{l}(\boldsymbol{\Gamma}, k)\right] .
$$

Let $\bar{h}_{l}(k)=\bar{y}_{l}(k)-\bar{g}_{l}(\boldsymbol{\Gamma}, k)$ and $\tilde{h}_{l}(k)=\tilde{y}_{l}(k)-\tilde{g}_{l}(\boldsymbol{\Gamma}, k)$, and then, we obtain

$$
\begin{aligned}
& \frac{\partial}{\partial \Gamma_{i}} f_{h}\left[\bar{y}_{l}(k)-\bar{g}_{l}(\boldsymbol{\Gamma}, k), \tilde{y}_{l}(k)-\tilde{g}_{l}(\boldsymbol{\Gamma}, k)\right] \\
& =\left.\frac{\partial}{\partial \bar{h}} f_{h}(\bar{h}, \tilde{h})\right|_{\left(\bar{h}_{l}(k), \tilde{h}_{l}(k)\right)} \cdot\left[-\frac{\partial \bar{g}_{l}(\boldsymbol{\Gamma}, k)}{\partial \Gamma_{i}}\right] \\
& \quad+\left.\frac{\partial}{\partial \tilde{h}_{h}} f_{h}(\bar{h}, \tilde{h})\right|_{\left(\bar{h}_{l}(k), \tilde{h}_{l}(k)\right)} \cdot\left[-\frac{\partial \tilde{g}_{l}(\boldsymbol{\Gamma}, k)}{\partial \Gamma_{i}}\right],
\end{aligned}
$$

and

$$
\begin{aligned}
J_{i j}= & I_{\mathrm{r}}(\boldsymbol{\Omega}) \sum_{k=1}^{K} \sum_{l=1}^{M N} \frac{\partial \bar{g}_{l}(\boldsymbol{\Gamma}, k)}{\partial \Gamma_{i}} \frac{\partial \bar{g}_{l}(\boldsymbol{\Gamma}, k)}{\partial \Gamma_{j}} \\
& +I_{\mathrm{i}}(\boldsymbol{\Omega}) \sum_{k=1}^{K} \sum_{l=1}^{M N} \frac{\partial \tilde{g}_{l}(\boldsymbol{\Gamma}, k)}{\partial \Gamma_{i}} \frac{\partial \tilde{g}_{l}(\boldsymbol{\Gamma}, k)}{\partial \Gamma_{j}},
\end{aligned}
$$

where

$$
\begin{aligned}
& I_{\mathrm{r}}(\boldsymbol{\Omega})=E\left\{\left[\frac{\frac{\partial}{\partial \tilde{h}_{h}}(\bar{h}, \tilde{h})}{f_{h}(\bar{h}, \tilde{h})}\right]^{2}\right\}, \\
& I_{\mathrm{i}}(\boldsymbol{\Omega})=E\left\{\left[\frac{\frac{\partial}{\partial \tilde{h}} f_{h}(\bar{h}, \tilde{h})}{f_{h}(\bar{h}, \tilde{h})}\right]^{2}\right\} .
\end{aligned}
$$

We assume that the $\operatorname{PDF} f_{h}(\bar{h}, \tilde{h})$ is circularly symmetric, and thus, $f_{h}( \pm \bar{h}, \pm \tilde{h})=f_{h}(\bar{h}, \tilde{h})=f\left(\sqrt{\bar{h}^{2}+\tilde{h}^{2}}\right)$, which demonstrates that $\bar{h}_{l}(k)$ and $\tilde{h}_{l}(k)$ are uncorrelated with zero-mean. Then, $I_{\mathbf{r}}(\boldsymbol{\Omega})$ and $I_{\mathbf{i}}(\boldsymbol{\Omega})$ are equivalent to

$$
I_{\mathbf{c}}(\boldsymbol{\Omega})=\pi \int_{0}^{\infty} \frac{\left[f^{\prime}(\xi)\right]^{2}}{f(\xi)} \xi d \xi
$$

In this case, we can obtain

$J_{i j}=I_{\mathrm{c}}(\boldsymbol{\Omega}) \sum_{k=1}^{K} \operatorname{Re}\left[\left(\frac{\partial}{\partial \Gamma_{i}} \mathbf{g}(\boldsymbol{\Gamma}, k)\right)^{H}\left(\frac{\partial}{\partial \Gamma_{j}} \mathbf{g}(\boldsymbol{\Gamma}, k)\right)\right]$,

where $\left[\left(\partial / \partial \Gamma_{i}\right) \mathbf{g}(\boldsymbol{\Gamma}, k)\right]=\left[\left(\partial / \partial \Gamma_{i}\right) g_{1}(\boldsymbol{\Gamma}, k),\left(\partial / \partial \Gamma_{i}\right) g_{2}(\boldsymbol{\Gamma}, k)\right.$, $\left.\ldots,\left(\partial / \partial \Gamma_{i}\right) g_{M N}(\boldsymbol{\Gamma}, k)\right]^{T}$.

We assume that $N_{\text {pa }}$ denotes the number of parameters in $\boldsymbol{\Gamma}$, and thus, we can obtain the matrix $[(\partial / \partial \boldsymbol{\Gamma}) \mathbf{g}(\boldsymbol{\Gamma}, k)]=\left[\left(\partial / \partial \Gamma_{1}\right) \mathbf{g}(\boldsymbol{\Gamma}, k),\left(\partial / \partial \Gamma_{2}\right) \mathbf{g}(\boldsymbol{\Gamma}, k), \ldots\right.$,

$\left.\left(\partial / \partial \Gamma_{N_{\mathrm{pa}}}\right) \mathbf{g}(\boldsymbol{\Gamma}, k)\right]$. Then, the FIM is represented as follows:

$$
\mathbf{J}_{\boldsymbol{\Gamma}}=I_{\mathrm{c}}(\boldsymbol{\Omega}) \sum_{k=1}^{K} \operatorname{Re}\left[\left(\frac{\partial}{\partial \boldsymbol{\Gamma}} \mathbf{g}(\boldsymbol{\Gamma}, k)\right)^{H}\left(\frac{\partial}{\partial \Gamma} \mathbf{g}(\boldsymbol{\Gamma}, k)\right)\right] .
$$

Finally, the CRB can be obtained by calculating the inverse of $\mathbf{J}_{\boldsymbol{\Gamma}}$ as follows [30]:

$$
\mathrm{CRB}(\boldsymbol{\Theta})=\frac{\left\{\operatorname{Re}\left[\left(\mathbf{D}^{H} \mathbf{P}_{\mathbf{C}}^{\perp} \mathbf{D}\right) \oplus\left(\mathbf{R}_{\beta}^{T} \otimes \mathbf{1}_{2 \times 2}\right)\right]\right\}^{-1}}{I_{\mathrm{C}}(\boldsymbol{\Omega})},
$$

where $\boldsymbol{\Theta}=\left[\theta_{1}, \varphi_{1}, \theta_{2}, \varphi_{2}, \ldots, \theta_{P}, \varphi_{P}\right]^{T}, \mathbf{D}=\left[\mathbf{d}\left(\theta_{1}\right)\right.$, $\left.\mathbf{d}\left(\varphi_{1}\right), \mathbf{d}\left(\theta_{2}\right), \mathbf{d}\left(\varphi_{2}\right), \ldots, \mathbf{d}\left(\theta_{P}\right), \mathbf{d}\left(\varphi_{P}\right)\right]$ with $\mathbf{d}\left(\theta_{i}\right)=\partial\left[\boldsymbol{\alpha}_{\mathrm{t}}\left(\theta_{i}\right)\right.$ $\left.\otimes \boldsymbol{\alpha}_{\mathrm{r}}\left(\varphi_{i}\right)\right] / \partial\left(\theta_{i}\right)$ and $\mathbf{d}\left(\varphi_{i}\right)=\partial\left[\boldsymbol{\alpha}_{\mathrm{t}}\left(\theta_{i}\right) \otimes \boldsymbol{\alpha}_{\mathrm{r}}\left(\varphi_{i}\right)\right] / \partial\left(\varphi_{i}\right)$, $\mathbf{P}_{\mathbf{C}}^{\perp}=\mathbf{I}-\mathbf{P}_{\mathbf{C}}$ denotes the projection onto the orthogonal complementary space of $\mathbf{C}, \mathbf{R}_{\beta}=1 / K \sum_{k=1}^{K} \boldsymbol{\beta}(k) \boldsymbol{\beta}^{H}(k)$, and $\mathbf{1}_{2 \times 2}$ denotes a $2 \times 2$ matrix covered with ones.

To summarize, the CRB can be written as a product of two terms. The first term depends on the noise, and the second term depends on the signal. For the first term, the $\operatorname{PDF} f(\xi)$ is a major issue of interest for $I_{\mathrm{C}}(\Omega)$. Usually, the $\operatorname{PDF} f(\xi)$ is obtained via the inverse Fourier transform of the characteristic function [10]. Nevertheless, the closed-form expression of $f(\xi)$ does not exist, except for $\alpha=1$ and $\alpha=2$ (Cauchy and Gaussian distributions, respectively) [10], which has become an obstacle to obtaining the CRB. 
In this context, the Cauchy-Gaussian mixture (CGM) model [31] is an appropriate approximation model for the $\mathrm{S} \alpha \mathrm{S}$ PDF. For the purpose of reducing the computational burden in the CGM model, we adopt the bi-parameter CGM (BCGM) model [31] to obtain the PDF as follows:

$$
\begin{aligned}
f(\xi) & =(1-\chi) f_{\mathrm{G}}(\xi)+\chi f_{\mathrm{C}}(\xi) \\
& =(1-\chi) \frac{1}{2 \gamma \sqrt{\pi}} \exp \left(-\frac{\xi^{2}}{4 \gamma^{2}}\right)+\chi \frac{\gamma}{\pi\left(\xi^{2}+\gamma^{2}\right)},
\end{aligned}
$$

where $\chi$ denotes the mixture ratio, and $\gamma$ represents the scale. In this way, we can obtain the CRB in the impulse noise through Eqs. (31) and (34).

\section{Simulation results and discussion}

For the purpose of evaluating the direction-finding performance of QGWO-IN-WSSF, a chain of experiments will be conducted in this section. We consider the ULAs of six transmitting elements and six receiving elements with half-wavelength inter-element spacing in bistatic MIMO radar. For the QGWO, the size of the population $Q=$ 30 , and the number of iterations is set to 100 . To make the simulation results more reliable, 500 Monte-Carlo experiments are performed for each scenario.

In the following, the performances of the QGWO-INWSSF, FLOM-MUSIC (the fractional order is set to 1.2) [14], IN-MUSIC [14], and the CRB, are compared.

\subsection{First scenario}

First, we consider two independent sources located at $\left(\theta_{1}, \varphi_{1}\right)=\left(30^{\circ}, 40^{\circ}\right),\left(\theta_{2}, \varphi_{2}\right)=\left(36^{\circ}, 46^{\circ}\right)$. To assess the estimation accuracy, we define the root mean square error (RMSE) as follows:

$$
\text { RMSE }=\sqrt{\sum_{p=1}^{P} \sum_{\dot{n}=1}^{N_{\mathrm{ex}}} \frac{\left[\left(\theta_{p}-\hat{\theta}_{p \dot{n}}\right)^{2}+\left(\varphi_{p}-\hat{\varphi}_{p \dot{n}}\right)^{2}\right]}{2 P N_{\mathrm{ex}}}},
$$

where $P$ is the number of sources, $N_{\mathrm{ex}}$ is the number of Monte-Carlo experiments, $\theta_{p}$ and $\varphi_{p}$ denote the true value of DOD and DOA for the $p$ th source, respectively, and $\hat{\theta}_{p \dot{n}}$ and $\hat{\varphi}_{p \dot{n}}$ denote the estimated value of DOD and DOA for the $p$ th source in the $\dot{n}$ th experiment, respectively.

For the purpose of assessing the performance of QGWO-IN-WSSF under the circumstances of different numbers of snapshots, the RMSE curves and the CRB of two independent sources are displayed for different numbers of snapshots in Fig. 1 with GSNR $=20 \mathrm{~dB}, \alpha=1.5$, $\chi=0.4, \gamma=1$. It is shown that the RMSE of the proposed QGWO-IN-WSSF method is quite close to the CRB asymptotically, which indicates that it can obtain an accurate estimation of two independent sources in the impulse noise. In addition, the proposed QGWO-INWSSF method outperforms the existing FLOM-MUSIC method and IN-MUSIC method in the matter of estimation accuracy in such a small-snapshot domain.

In Fig. 2, the success rate curves of two independent sources are exhibited for different numbers of snapshots with GSNR $=20 \mathrm{~dB}, \alpha=1.5$. Basically, we consider that an angle is successfully estimated when the angular distance between the estimated value and the true value becomes smaller than $1^{\circ}$, and the success rate indicates the percentage of successful estimations. As we can observe in

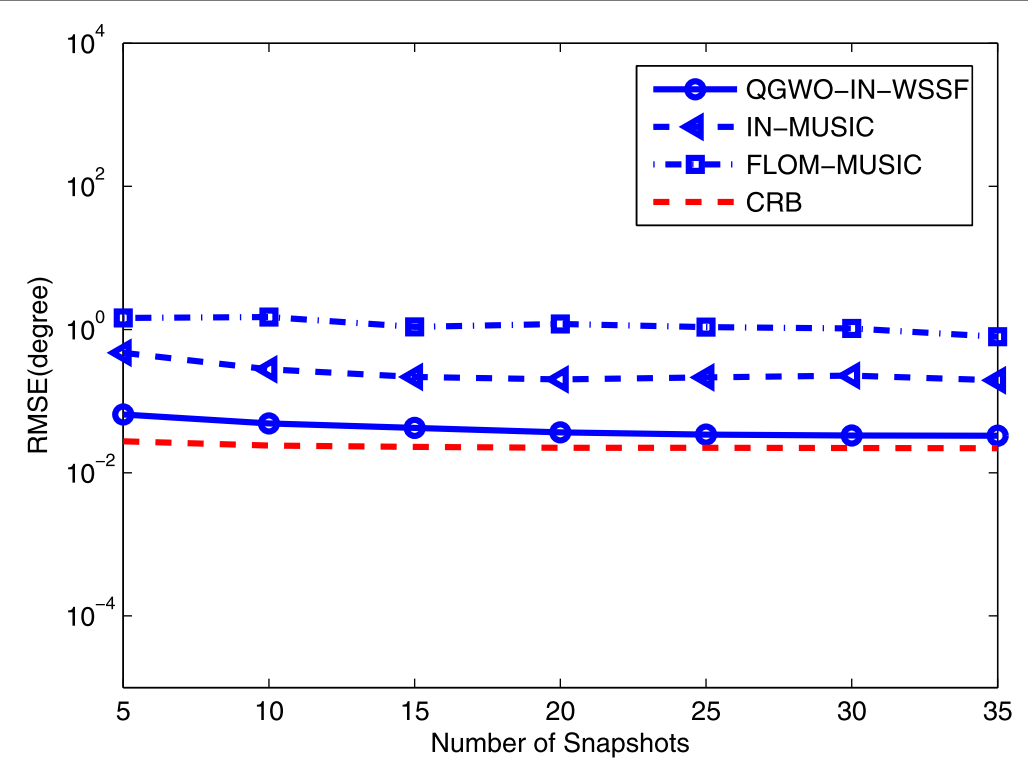

Fig. 1 RMSE curves and CRB versus the number of snapshots for two independent sources with GSNR=20dB and $\alpha=1.5$ 


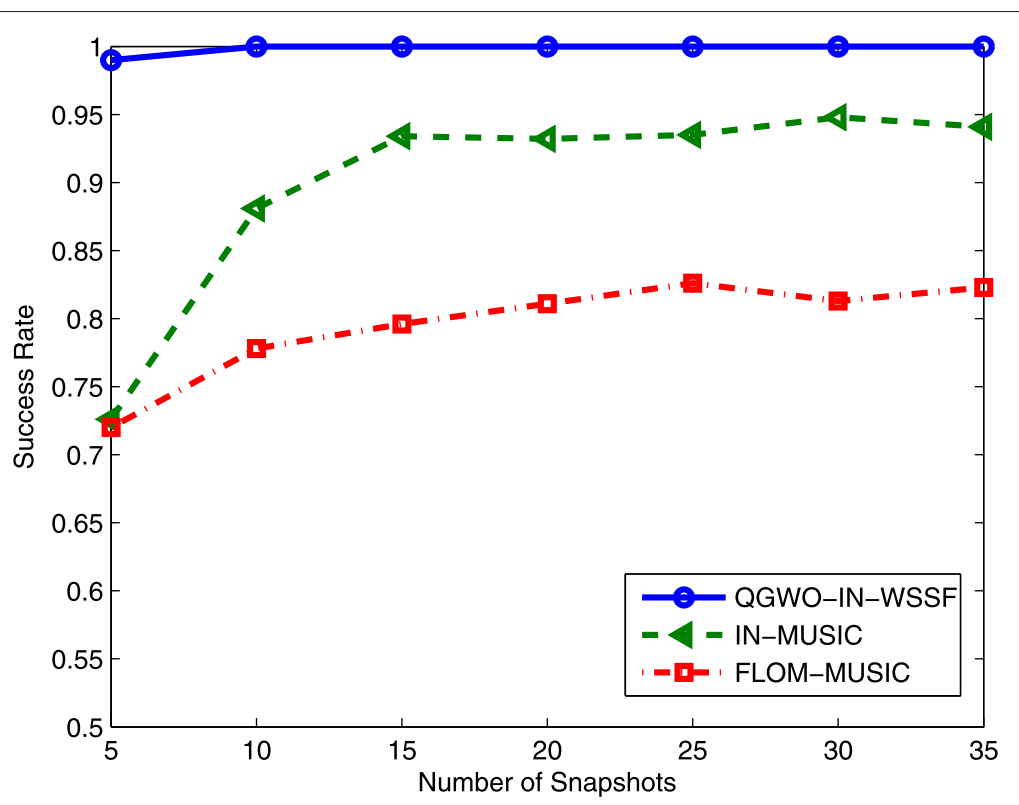

Fig. 2 Success rate curves versus the number of snapshots for two independent sources with GSNR=20dB and $\alpha=1.5$

Fig. 2, the proposed QGWO-IN-WSSF method can successfully estimate the DODs and DOAs of the targets in almost all of the experiments with a small number of snapshots. Moreover, the proposed QGWO-IN-WSSF method is better than the existing FLOM-MUSIC method and INMUSIC method in the matter of success rate, especially in the small-snapshot domain, which demonstrates that the QGWO-IN-WSSF is robust for direction finding with a small number of snapshots.

\subsection{Second scenario}

Next, we consider the same two independent sources, and the number of snapshots $K$ is set to 20 with the identical simulation parameters as in the first scenario.

Based on the IN-WSSF function, Fig. 3 exhibits the performance of QGWO and a number of conventional intelligence algorithms, such as ABC [17], HS [18], CS [19], and GWO [23]. The IN-WSSF methods based on the $\mathrm{ABC}, \mathrm{HS}, \mathrm{CS}$, and GWO are referred to as $\mathrm{ABC}-\mathrm{IN}-\mathrm{WSSF}$,

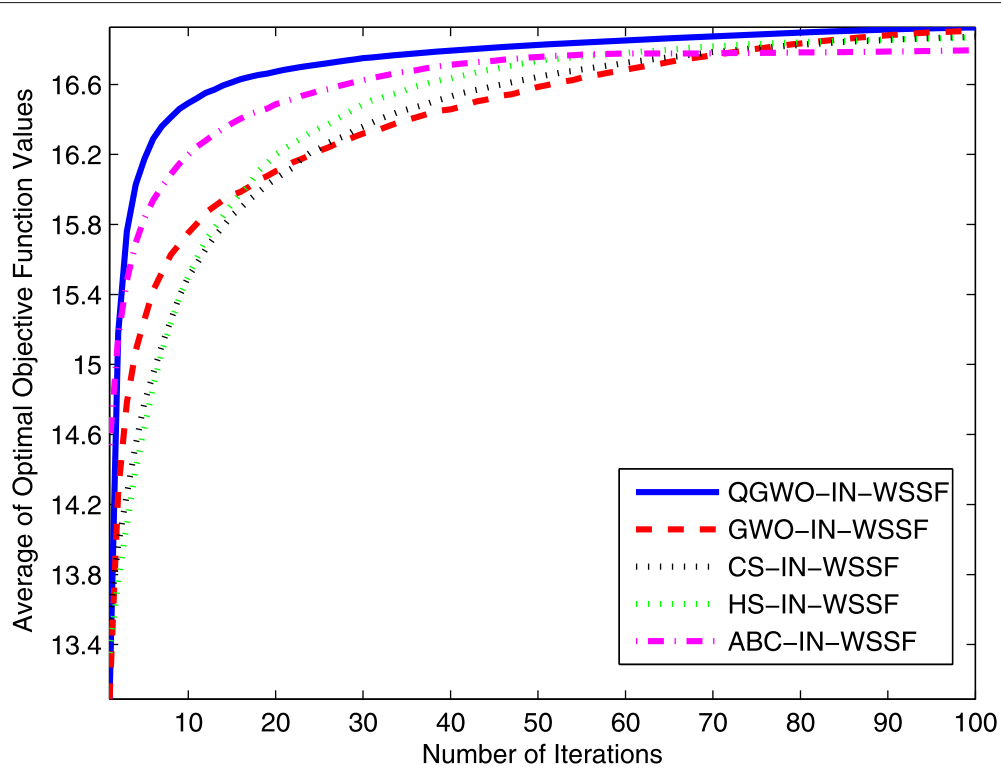

Fig. 3 Convergence curves of five intelligence algorithms for two independent sources with GSNR=20dB and $\alpha=1.5$ 
HS-IN-WSSF, CS-IN-WSSF, and GWO-IN-WSSF for short, respectively. The size of the population is 30 , and the number of iterations is 100 in all of the intelligence algorithms. Additionally, the result shown in Fig. 3 demonstrates that QGWO is better than $\mathrm{ABC}, \mathrm{HS}, \mathrm{CS}$, and GWO according to the speed and accuracy of convergence, which indicates the superiority of QGWO.

In Fig. 4, the RMSE curves and the CRB of two independent sources are displayed for different GSNRs with $\alpha=1.5$. As we can observe in Fig. 4, the RMSE of the proposed QGWO-IN-WSSF method is quite close to the CRB asymptotically. The RMSE of the three methods and the CRB worsen with a decrease in the GSNR because the lower GSNR shifts the estimated values from the true values in such a way that the RMSE is more pronounced. Moreover, the proposed QGWO-IN-WSSF method outperforms the FLOM-MUSIC method and INMUSIC method in the matter of estimation accuracy.

In Fig. 5, the success rate curves of two independent sources are shown for different characteristic exponents with GSNR $=20 \mathrm{~dB}$. As we can observe in Fig. 5, the proposed QGWO-IN-WSSF method can successfully estimate DODs and DOAs of targets in almost all of the experiments in the weak impulse noise. Moreover, as the characteristic exponent increases, the success rate of the three methods rises apparently. In addition, the proposed QGWO-IN-WSSF method is better than the FLOM-MUSIC method and IN-MUSIC method in the matter of success rate, especially in the strong impulse noise.

\subsection{Third scenario}

To examine whether the different angles affect the performance of the proposed QGWO-IN-WSSF method, we consider another two independent sources located at $\left(\theta_{1}, \varphi_{1}\right)=\left(30^{\circ}, 40^{\circ}\right),\left(\theta_{2}, \varphi_{2}\right)=\left(40^{\circ}, 50^{\circ}\right)$, and the other simulation parameters are identical to those in the second scenario. Then, simulations were performed to calculate the RMSE and success rate, respectively.

The RMSE curves, as well as the CRB of two different independent sources, are exhibited for different GSNRs with $\alpha=1.5$ in Fig. 6, and the success rate curves of two different independent sources are shown for different characteristic exponents, with GSNR $=20 \mathrm{~dB}$ in Fig. 7. We can obtain some conclusions that are similar to those in the second scenario from Figs. 6 and 7. In addition, the larger difference in the angles makes the direction finding easier.

Hence, we can conclude that the proposed QGWO-INWSSF method can obtain the desired performance for different angles.

\subsection{Fourth scenario}

The previous simulations were conducted for only two sources. For the purpose of testing whether the performance of QGWO-IN-WSSF is influenced by the increasing number of sources, we consider three independent sources located at $\left(\theta_{1}, \varphi_{1}\right)=\left(30^{\circ}, 40^{\circ}\right),\left(\theta_{2}, \varphi_{2}\right)=$ $\left(40^{\circ}, 50^{\circ}\right),\left(\theta_{3}, \varphi_{3}\right)=\left(50^{\circ}, 60^{\circ}\right)$, and the other simulation parameters are identical to those in the third scenario.

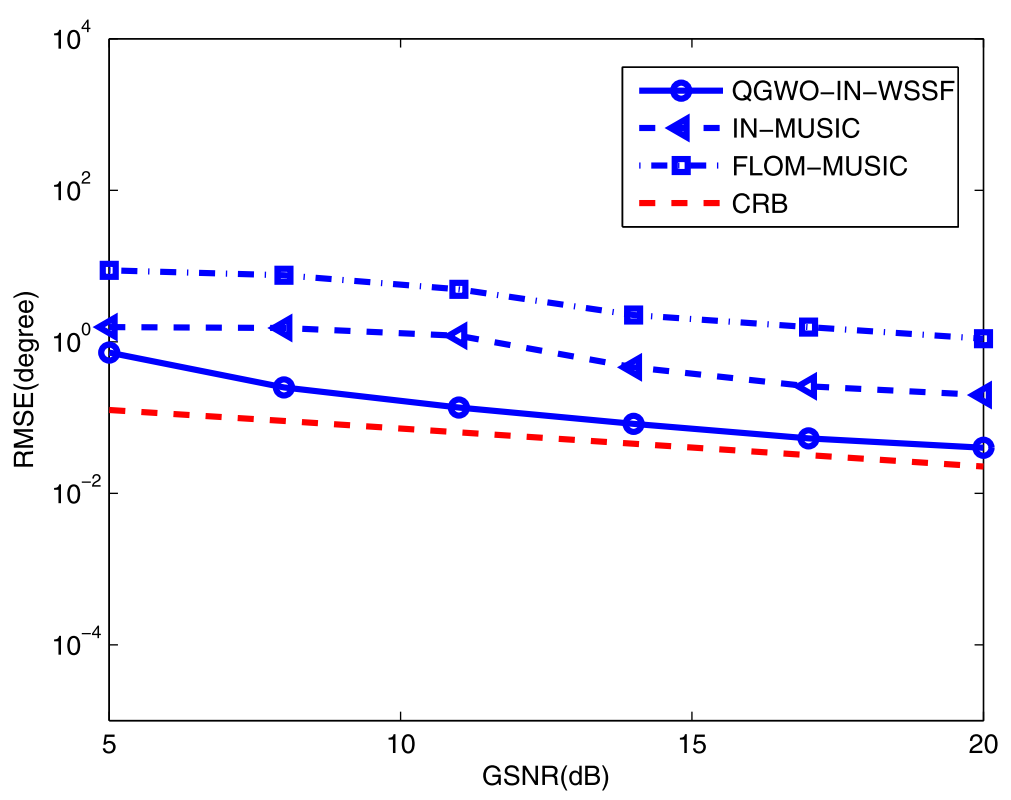

Fig. 4 RMSE curves and CRB versus GSNR for two independent sources with $\alpha=1.5$ 


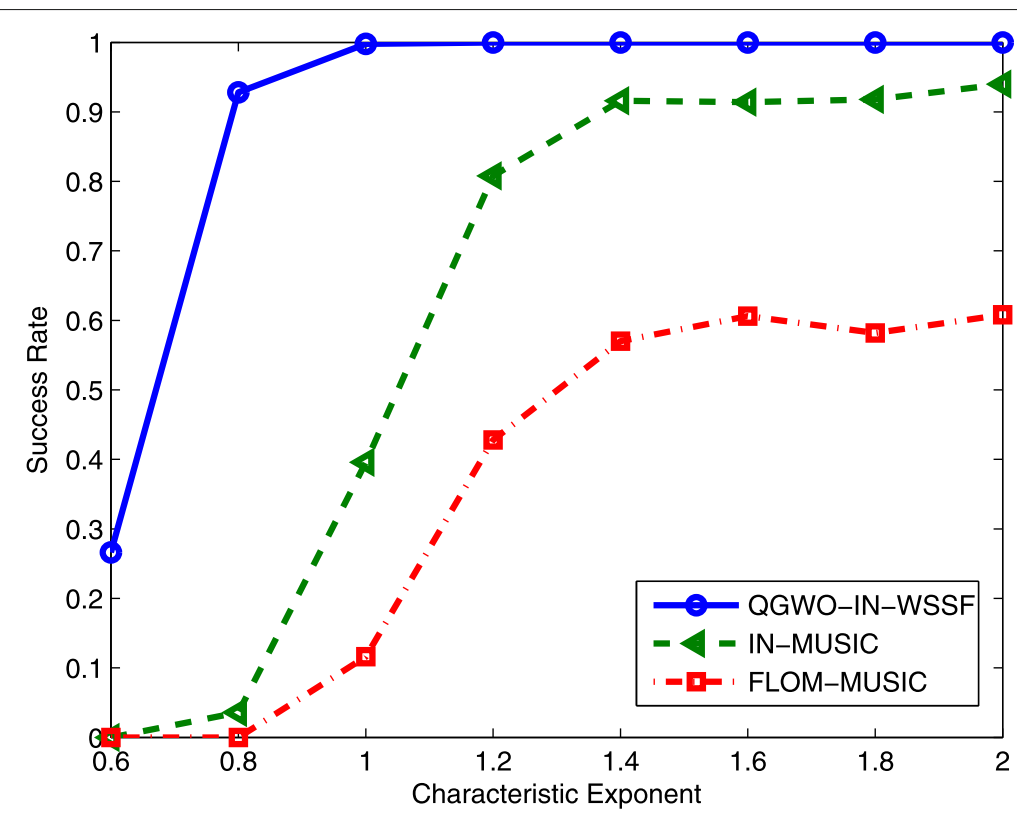

Fig. 5 Success rate curves versus characteristic exponent for two independent sources with GSNR $=20 \mathrm{~dB}$

In Fig. 8, the RMSE curves, as well as the CRB of three independent sources, are displayed for different GSNRs with $\alpha=1.5$. In Fig. 9, the success rate curves of three independent sources are exhibited for different characteristic exponents with GSNR $=20 \mathrm{~dB}$. From Figs. 8 and 9, we can conclude that the proposed QGWO-IN-WSSF method has the capacity to locate the DODs and DOAs precisely with the increase in the number of sources.
Additionally, the other conclusions are similar to those in the previous experiments.

\subsection{Fifth scenario}

The previous simulations are performed only for some scenarios of independent sources. However, the coherent sources will present some challenges for direction finding. In this sense, the performance of the FLOM-MUSIC

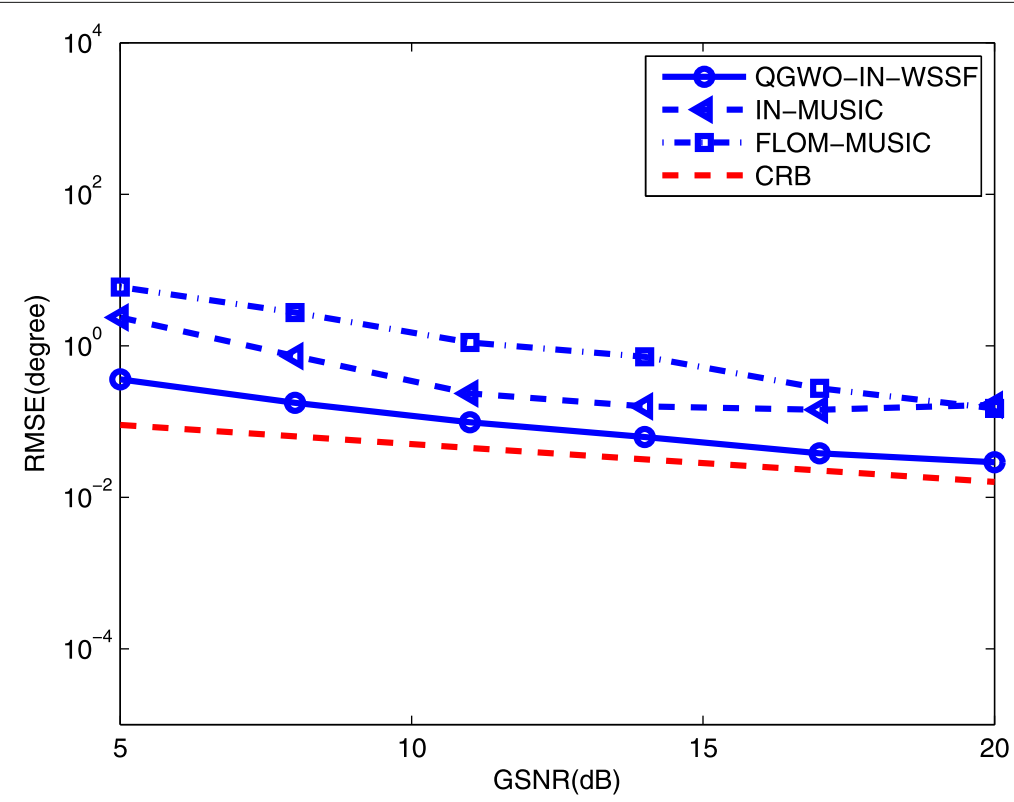

Fig. 6 RMSE curves and CRB versus GSNR for two different independent sources with $\alpha=1.5$ 


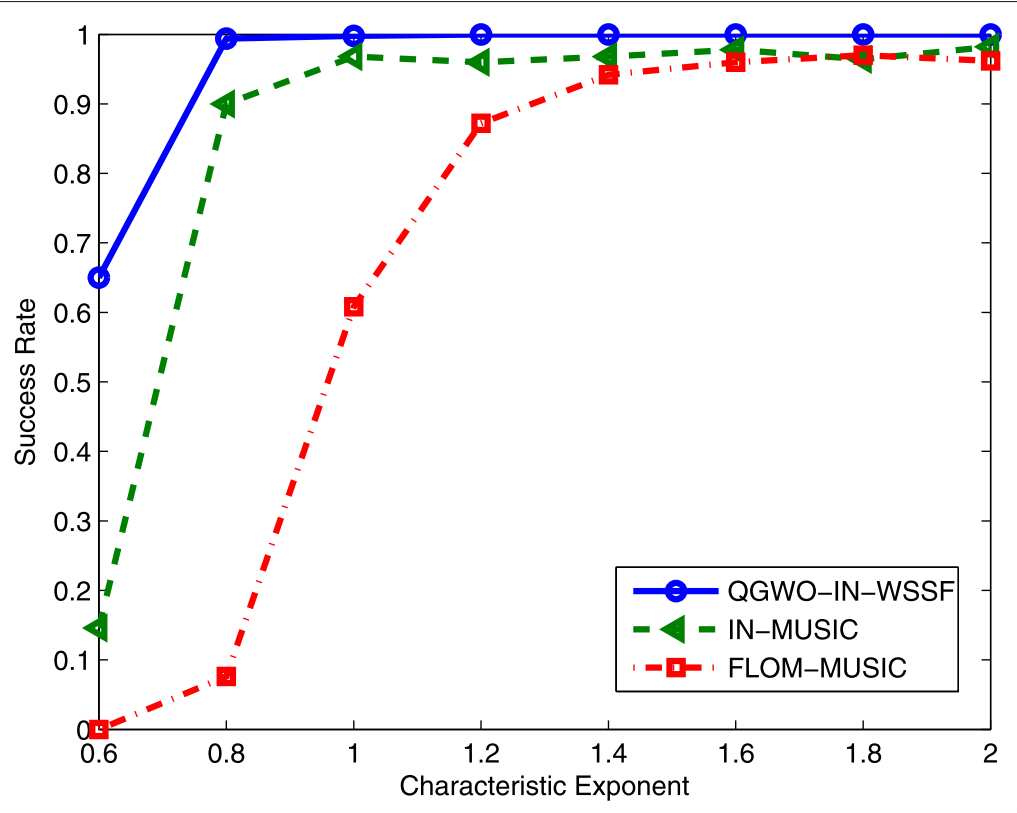

Fig. 7 Success rate curves versus characteristic exponent for two different independent sources with GSNR=20dB

method and IN-MUSIC method will deteriorate severely when locating the coherent sources [32]. Hence, we employ forward-backward spatial smoothing (SS) to handle the coherent sources [33, 34].

For the purpose of examining the capacity of the proposed QGWO-IN-WSSF method to locate the coherent sources, two coherent sources located at $\left(\theta_{1}, \varphi_{1}\right)=$ $\left(30^{\circ}, 40^{\circ}\right),\left(\theta_{2}, \varphi_{2}\right)=\left(40^{\circ}, 50^{\circ}\right)$ are considered, and the other simulation parameters are identical to those in the third scenario. Then, the simulations have been performed to obtain the RMSE and success rate.

The RMSE curves and the CRB of two coherent sources are displayed for different GSNRs with $\alpha=1.5$ in Fig. 10, and the success rate curves of two coherent sources are exhibited for different characteristic exponents with GSNR $=20 \mathrm{~dB}$ in Fig. 11. From Figs. 10 and 11 , we can conclude that the proposed QGWO-IN-WSSF method is capable of locating the DODs and DOAs of the

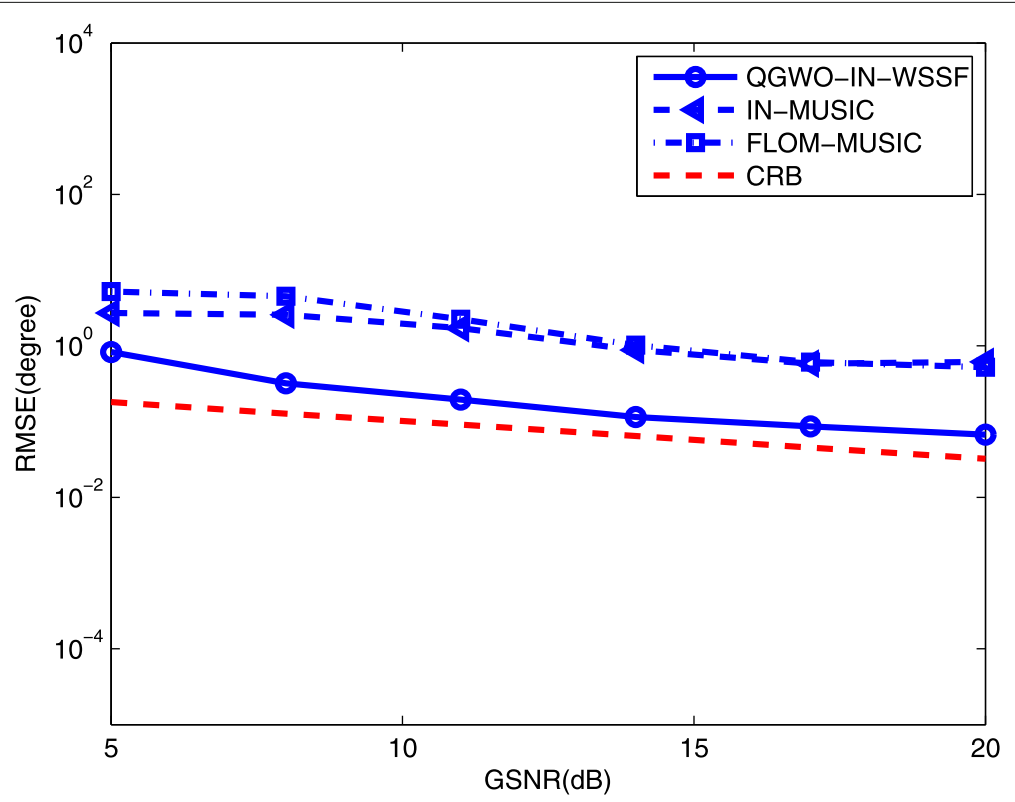

Fig. 8 RMSE curves and CRB versus GSNR for three independent sources with $\alpha=1.5$ 


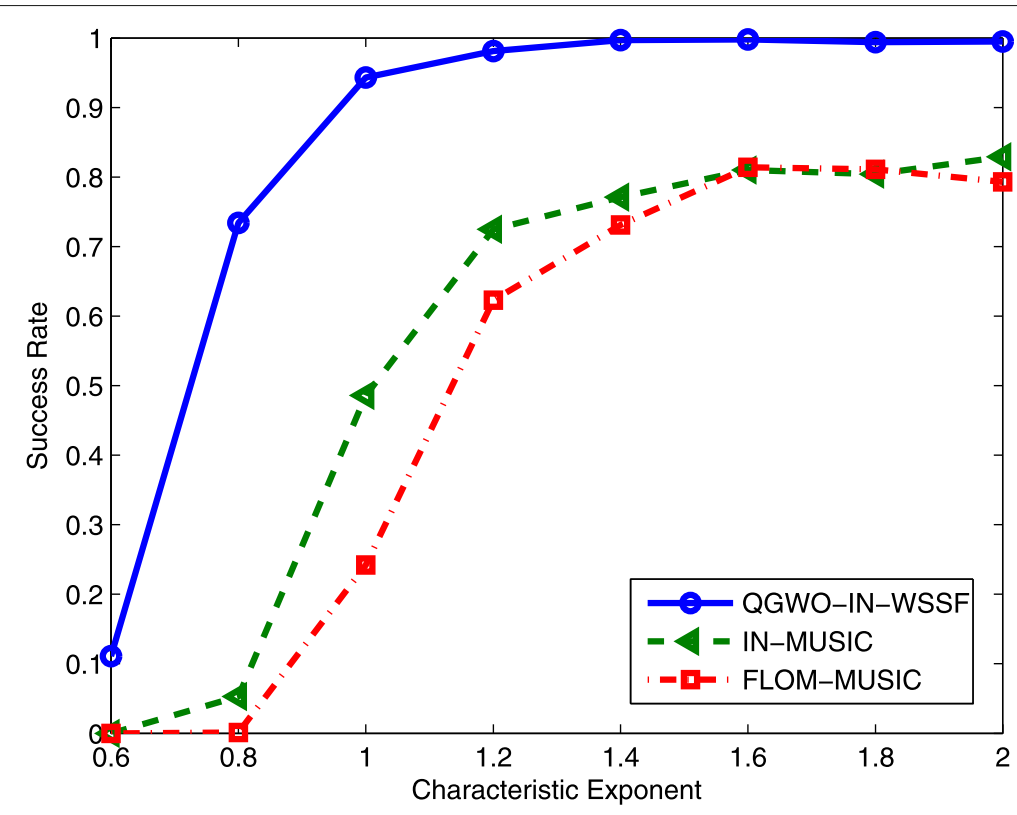

Fig. 9 Success rate curves versus characteristic exponent for three independent sources with GSNR=20dB

two coherent sources accurately. Moreover, the proposed QGWO-IN-WSSF method has obtained the desired performance again when locating the coherent sources, and it outperforms the FLOM-SSMUSIC method and IN-SSMUSIC method according to the estimation accuracy and success rate.

As a consequence, the performance of the proposed QGWO-IN-WSSF method does not worsen when addressing the coherent sources, which testifies to the robustness and superiority of the method. In this way, it is worthwhile to apply this method for locating the coherent sources.

\section{Conclusions}

In this paper, a novel method referred to as QGWOIN-WSSF is presented for locating the targets of bistatic MIMO radar in the impulse noise. A chain of MonteCarlo experiments has demonstrated that the proposed QGWO-IN-WSSF method can suppress the noise and locate multiple independent and coherent sources

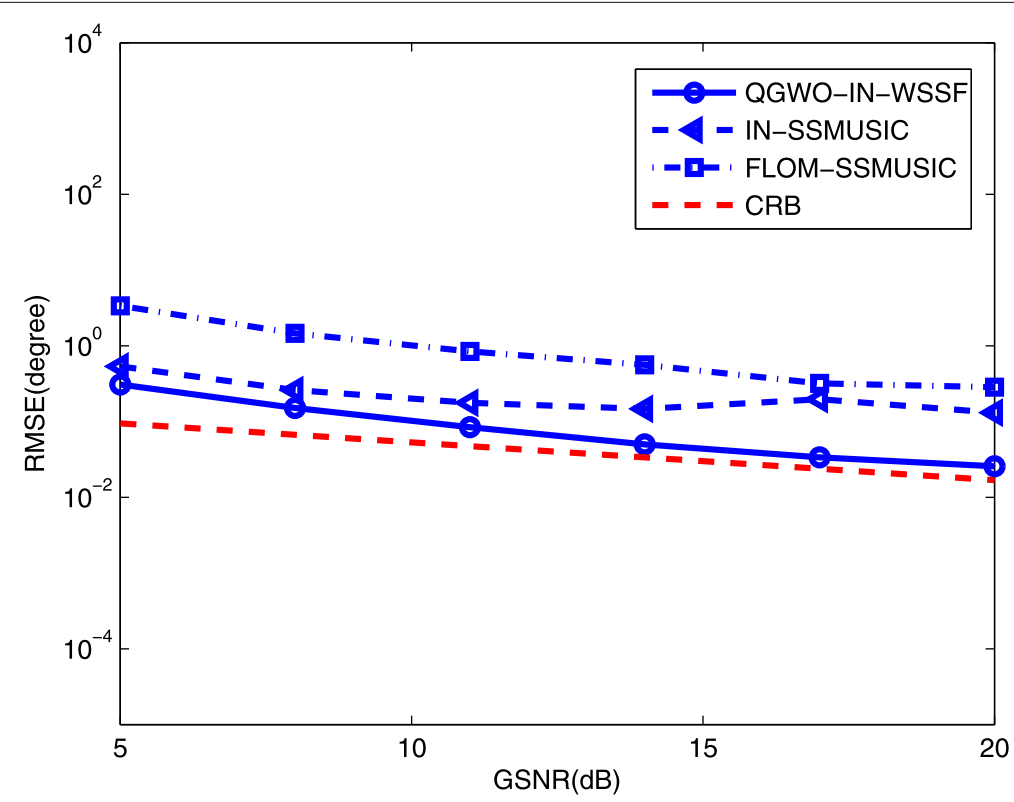

Fig. 10 RMSE curves and CRB versus GSNR for two coherent sources with $\alpha=1.5$ 


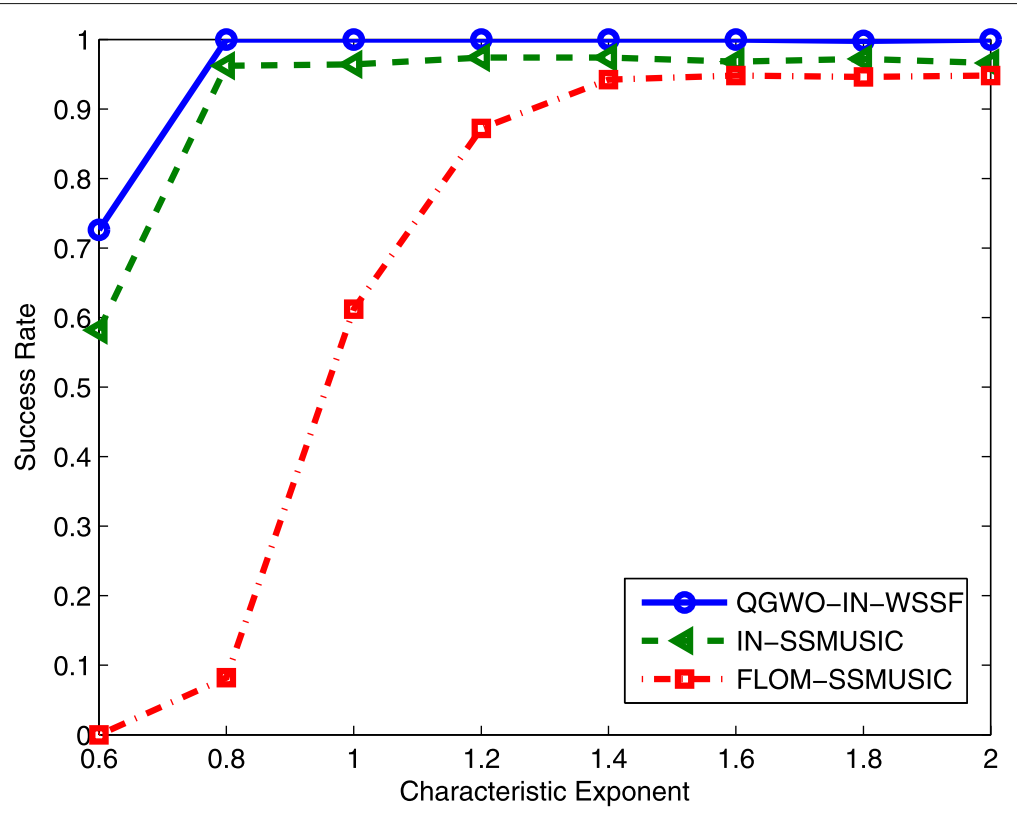

Fig. 11 Success rate curves versus characteristic exponent for two coherent sources with GSNR=20dB

effectively. Compared with some previous methods, the proposed QGWO-IN-WSSF method is capable of obtaining better performance with a small number of snapshots, which verifies the superiority and robustness of the method. In addition, we derive a general CRB, which generalizes the Gaussian CRB, for direction finding of bistatic MIMO radar in the impulse noise. In the subsequent research, we will design a multimodal QGWO algorithm to solve more complicated direction-finding problems for MIMO radar.

\section{Appendix}

Proof Because the component $\left[\mathbf{R}_{\mathrm{z}}\right]_{i j}(i, j=1,2, \ldots, M N)$ of $\mathbf{R}_{\mathrm{z}}$ is a complex number, we should demonstrate that both real part $\operatorname{Re}\left\{\left[\mathbf{R}_{\mathbf{z}}\right]_{i j}\right\}$ and imaginary part $\operatorname{Im}\left\{\left[\mathbf{R}_{\mathbf{z}}\right]_{i j}\right\}$ are bounded. Thus, we can obtain

$$
\begin{aligned}
\operatorname{Re}\left\{\left[\mathbf{R}_{\mathrm{z}}\right]_{i j}\right\} & =\operatorname{Re}\left\{E\left[z_{i}(k) z_{j}^{*}(k)\right]\right\} \\
& =E\left\{\operatorname{Re}\left[z_{i}(k) z_{j}^{*}(k)\right]\right\} \\
& \leq E\left\{\left|z_{i}(k) z_{j}^{*}(k)\right|\right\} \\
& \leq E\left\{\left|z_{i}(k)\right|\left|z_{j}(k)\right|\right\} \\
& =E\left\{\frac{\left|y_{i}(k)\right|}{\max \left\{\left|y_{1}(k)\right|,\left|y_{2}(k)\right|, \ldots,\left|y_{M N}(k)\right|\right\}}\right. \\
& \left.\times \frac{\left|y_{j}(k)\right|}{\max \left\{\left|y_{1}(k)\right|,\left|y_{2}(k)\right|, \ldots,\left|y_{M N}(k)\right|\right\}}\right\} \\
& \leq E\left\{\frac{y_{i}(k)}{y_{i}(k)}|\times| \frac{y_{j}(k)}{y_{j}(k)} \mid\right\}=1 .
\end{aligned}
$$

Note that

$$
\begin{aligned}
\operatorname{Re}\left\{\left[\mathbf{R}_{\mathrm{z}}\right]_{i j}\right\} & \geq-E\left\{\left|z_{i}(k) z_{j}^{*}(k)\right|\right\} \\
& \geq-E\left\{\left|z_{i}(k)\right|\left|z_{j}(k)\right|\right\} \\
& \geq-1 .
\end{aligned}
$$

In this case, we can obtain $-1 \leq \operatorname{Re}\left\{\left[\mathbf{R}_{\mathrm{z}}\right]_{i j}\right\} \leq 1$. Similarly, $-1 \leq \operatorname{Im}\left\{\left[\mathbf{R}_{\mathrm{z}}\right]_{i j}\right\} \leq 1$. Hence, weighted signal covariance matrix $\mathbf{R}_{\mathrm{Z}}$ is bounded in the impulse noise.

\section{Abbreviations}

ABC: Artificial bee colony; BCGM: Bi-parameter Cauchy-Gaussian mixture; CGM: Cauchy-Gaussian mixture; CRB: Cramér-Rao bound; CS: Cuckoo search; DOD: Direction of departure; DOA: Direction of arrival; FIM: Fisher information matrix FLOM: Fractional low-order moment; GSNR: Generalized signal-to-noise ratio; GWO: Grey wolf optimization; HS: Harmony search; IN: Infinite norm; MIMO: Multiple-input-multiple-output; PDF: Probability distribution function; PSO: Particle swarm optimization; QGWO: Quantum-inspired grey wolf optimization; Root-MUSIC: Root multiple signal classification; RMSE: Root mean square error; S $\alpha$ S: Symmetric $\alpha$-stable; SNR: Signal-to-noise ratio; SS: Spatial smoothing; ULA: Uniform linear array; WSSF: Weighted signal subspace fitting

\section{Acknowledgements}

The authors would like to acknowledge the help of all the people who influenced the paper. Specifically, they would like to acknowledge the anonymous reviewers for their reasonable comments.

\section{Funding}

This work was sponsored by the National Natural Science Foundation of China (No. 61571149), the China Scholarship Council, the Fundamental Research Funds for the Central Universities (No. HEUCFP201808), the Special China Postdoctoral Science Foundation (No. 2015T80325), China Postdoctoral Science Foundation (No. 2013M530148), and Heilongjiang Postdoctoral Fund (No. LBH-Z13054).

Availability of data and materials Not applicable. 


\section{Authors' contributions}

HG conceived and designed the ideas and simulations. JL performed the simulations. JL and MD analyzed the data and results. JL wrote the manuscript. $\mathrm{HG}$ revised the manuscript. All authors read and approved the final manuscript.

\section{Competing interests}

The authors declare that they have no competing interests.

\section{Publisher's Note}

Springer Nature remains neutral with regard to jurisdictional claims in published maps and institutional affiliations.

Received: 21 April 2018 Accepted: 20 November 2018

Published online: 27 December 2018

\section{References}

1. J. Li, P. Stoica, L. Xu, W. Roberts, On parameter identifiability of MIMO radar. IEEE Sig. Process. Lett. 14(12), 968-971 (2007)

2. E. Fishler, A. Haimovich, R. S. Blum, L. J. Cimini, D. Chizhik, R. A. Valenzuela, Spatial diversity in radars-models and detection performance. IEEE Trans. Signal Process. 54(3), 823-838 (2006)

3. A. M. Haimovich, R. S. Blum, L. J. Cimini, MIMO radar with widely separated antennas. IEEE Signal Proc. Mag. 25(1), 116-129 (2008)

4. J. Li, P. Stoica, MIMO radar with colocated antennas. IEEE Signal Proc. Mag. 24(5), 106-114 (2007)

5. R. Boyer, Performance bounds and angular resolution limit for the moving colocated MIMO radar. IEEE Trans. Signal Process. 59(4), 1539-1552 (2011)

6. J. Li, X. Zhang, F. Wang, Quaternion root-MUSIC algorithm for angle estimation in bistatic MIMO radar. J. Electron. Inf. Technol. 34(2), 300-304 (2012)

7. Y. Guo, Y. Zhang, L. Zhang, N. Tong, Fast target localization and the mutual coupling self-calibration algorithm for bistatic MIMO radar. J. Xidian Univ. 39(5), 66-71 (2012)

8. G. Zheng, B. Chen, Unitary dual-resolution ESPRIT for joint DOD and DOA estimation in bistatic MIMO radar. Multidim. Syst. Sign. Process. 26(1), 159-178 (2015)

9. B. Tang, J. Tang, Y. Zhang, Z. Zheng, Maximum likelihood estimation of DOD and DOA for bistatic MIMO radar. Signal Process. 93(5), 1349-1357 (2013)

10. M. Shao, C. L. Nikias, Signal processing with fractional lower order moments: stable processes and their applications. Proc. IEEE. 81(7), 986-1010 (1993)

11. L. Boubchir, J. M. Fadili, A closed-form nonparametric Bayesian estimator in the wavelet domain of images using an approximate $\alpha$-stable prior. Pattern Recogn. Lett. 27(12), 1370-1382 (2006)

12. J. Gong, S. Lou, Y. Guo, W. Zhang, A target angle estimation method for bistatic MIMO radar in impulsive noise. Mod. Radar. 39(2), 29-32 (2017)

13. B. Liu, J. Zhang, X. Yuan, C. Xu, Z. Liu, Angle estimation for bistatic MIMO radar in impulsive noise environments. Xian Dianzi Keji Daxue Xuebao/J. Xidian Univ. 42(4), 182-187 (2015)

14. J. Wang, S. Jiang, J. He, Z. Liu, Subspace-based DOA estimation in impulsive noise environments for MIMO radars. J. Astronaut. 30(4), 1653-1657 (2009)

15. N. Hu, Z. Ye, D. Xu, S. Cao, A sparse recovery algorithm for DOA estimation using weighted subspace fitting. Signal Proc. 92(10), 2566-2570 (2012)

16. R. Poli, J. Kennedy, T. Blackwell, Particle swarm optimization. Swarm Intell. 1(1), 33-57 (2007)

17. D. Karaboga, B. Basturk, A powerful and efficient algorithm for numerical function optimization: artificial bee colony (ABC) algorithm. J. Glob. Optim. 39(3), 459-471 (2007)

18. Z. W. Geem, J. H. Kim, G. Loganathan, A new heuristic optimization algorithm: harmony search. Simul. Trans. Soc. Model. Simul. Int. 76(2), 60-68 (2001)

19. X. Yang, S. Deb, Engineering optimisation by cuckoo search. Int. J. Math. Model. Numer. Optimisation. 1(4), 330-343 (2010)

20. S. Yuan, S. Wang, N. Tian, Swarm intelligence optimization and its application in geophysical data inversion. Appl. Geophys. 6(2), 166-174 (2009)

21. I.-H. Kuo, S.-J. Horng, T.-W. Kao, et al., A hybrid swarm intelligence algorithm for the travelling salesman problem. Expert Syst. 27(3), 166-179 (2010)
22. R. Lahoz-Beltra, Quantum genetic algorithms for computer scientists. Computers. 5(4), 24 (2016)

23. S. Mirjalili, S. M. Mirjalili, A. Lewis, Grey wolf optimizer. Adv. Eng. Softw. 69 , 46-61 (2014)

24. H. Gao, C. Xu, C. Li, Quantum-inspired cultural bacterial foraging algorithm for direction finding of impulse noise. Int. J. Innov. Comput. Appl. 6(1), 44-54 (2014)

25. W.-J. Zeng, H.-C. So, L. Huang, $l_{p}$-MUSIC: robust direction-of-arrival estimator for impulsive noise environments. IEEE Trans. Signal Process. 61(17), 4296-4308 (2013)

26. D. Ciuonzo, G. Romano, R. Solimene, Performance analysis of time-reversal MUSIC. IEEE Trans. Signal Process. 63(10), 2650-2662 (2015)

27. D. Ciuonzo, P. Salvo Rossi, Noncolocated time-reversal MUSIC: high-SNR distribution of null spectrum. IEEE Signal Process. Lett. 24(4), 397-401 (2017)

28. Y. Noam, H. Messer, Notes on the tightness of the hybrid Cramér-Rao lower bound. IEEE Trans. Signal Process. 57(6), 2074-2084 (2009)

29. M. Ghogho, A. Swami, Fast computation of the exact FIM for deterministic signals in colored noise. IEEE Trans. Signal Process. 47(1), 52-61 (1999)

30. M. Vallisneri, Use and abuse of the Fisher information matrix in the assessment of gravitational-wave parameter-estimation prospects. Phys. Rev. D. 77(4), 73-76 (2007)

31. X. Li, J. Sun, L. Jin, M. Liu, Bi-parameter CGM model for approximation of $\alpha$-stable PDF. Electron. Lett. 44(18), 1096-1097 (2008)

32. R. Schmidt, Multiple emitter location and signal parameter estimation IEEE Trans. Antennas Propag. 34(3), 276-280 (1986)

33. S. U. Pillai, B. H. Kwon, Forward/backward spatial smoothing techniques for coherent signal identification. IEEE Trans. Acoust. Speech Signal Process. 37(1), 8-15 (1989)

34. Y.-H. Choi, On conditions for the rank restoration in forward/backward spatial smoothing. IEEE Trans. Signal Process. 50(11), 2900-2901 (2002)

\section{Submit your manuscript to a SpringerOpen ${ }^{\circ}$ journal and benefit from:}

- Convenient online submission

- Rigorous peer review

Open access: articles freely available online

- High visibility within the field

Retaining the copyright to your article

Submit your next manuscript at $>$ springeropen.com 